\title{
Synthesis, structural characterization and formation mechanism of giant-dielectric $\mathrm{CaCu}_{3} \mathrm{Ti}_{4} \mathrm{O}_{12}$ nanotubes
}

\author{
Nirupam Banerjee, Saluru Baba Krupanidhi* \\ Materials Research Center, Indian Institute of Science, Bangalore, India; nirupam@mrc.iisc.ernet.in; nirupam1986@gmail.com; \\ *Corresponding Author: sbk@mrc.iisc.ernet.in
}

Received 5 March 2010; revised 10 April 2010; accepted 18 April 2010.

\begin{abstract}
A capillary-enforced template-based method has been applied to fabricate calcium copper titanate $\left(\mathrm{CaCu}_{3} \mathrm{Ti}_{4} \mathrm{O}_{12}, \mathrm{CCTO}\right)$ nanotubes (diameter $\sim 200 \mathrm{~nm}$ ) by filling sol-gel CCTO precursor solution into the nanochannels of porous anodic aluminum oxide (AAO) templates, subsequent heating for phase formation and finally the removal of nano-channel templates by applying basic solution. X-ray diffraction (XRD), field emission scanning electron microscopy (FE-SEM) and transmission electron microscopy (TEM) equipped with Energy-dispersive $X$-ray spectroscopy (EDX) have been employed to characterize the morphology, structure, and composition of as-prepared nanotubes. XRD and selected-area electron diffraction (SAED) investigations demonstrated that postannealed $\left(750^{\circ} \mathrm{C}\right.$ for $\left.1 \mathrm{~h}\right)$ ССTO nanotubes were polycrystalline with a cubic pseudo-perovskite crystal structure. The FE-SEM and TEM results showed that ССТО nanotubes were of uniform diameter $(\sim 200 \mathrm{~nm})$ throughout their length. High resolution TEM (HRTEM) analysis confirmed that the obtained CСTO nanotubes are made of randomly aligned nano-particles 5-10 nm in size. EDX analysis demonstrated that stoichiometric $\mathrm{CaCu}_{3} \mathrm{Ti}_{4} \mathrm{O}_{12}$ was formed. The possible formation mechanism of CСТO nanotubes in the AAO template is discussed.
\end{abstract}

Keywords: Nano-Tubes; Sol-Gel; Templated Growth; High-K-Dielectrics

\section{INTRODUCTION}

Subsequent to the discovery of carbon nanotubes [1] by Iijima et al. many research groups around the globe have become involved in the development of one-dimensional nano-structures such as nano-tubes, nano-rods, nanowires etc. of various functional materials due to their important role in mesoscopic physics and nanoscale device fabrication, as they can be utilized as both nanoscale device elements and interconnections while retaining unique properties due to size confinement in the radial direction [2-6]. Recently efforts have been made to synthesize and understand the growth of different nanostructures of the functional materials such as complex dielectric and ferroelectric oxides because of their promising applications for pyroelectric detectors, piezoelectric actuators and transducers, nonvolatile memory, ultrasonic devices, piezoelectric ink-jet printers etc. [7-11]. Nanotubes of various materials have been synthesized through various methods, among which sol-gel template technology provides a versatile technique for synthesizing highly ordered and controlled with the same structure and properties at large-scale production of one dimensional nanostructures, which is the basic building block of nanodevices. The nanochannel of template can be viewed as a reactor, in which one-dimensional nanostructure of the desired material can be synthesized in which the shape and size of the nanostructures depend only on the dimension of the pores present in the template.

High dielectric constant materials are immensely useful for miniaturization of electronic devices which is a recent goal of our technological progress. In this scenario ferroelectric materials are on demand due to their high dielectric constant values. Hernandez et al. have fabricated $\mathrm{BaTiO}_{3}$ (BT) and $\mathrm{PbTiO}_{3}(\mathrm{PT})$ nanotubes using template approach [12]. Using the same synthetic approach Zhang et al. have fabricated highly ordered $\mathrm{Pb}\left(\mathrm{Zr}_{0.53} \mathrm{Ti}_{0.47}\right) \mathrm{O}_{3}$ (PZT) nanowires which were found to exhibit a significant piezoresponse and proposed for potential device applications [13]. Recently, Singh et al. reported the fabrication of $\mathrm{Ba}_{0.6} \mathrm{Sr}_{0.4} \mathrm{TiO}_{3}$ (BST) nanotubes [14]. But one serious drawback in these well known ferroelectric materials possessing higher dielectric constant values is these are $\mathrm{Pb}$ or $\mathrm{Ba}$ containing materials which are severely toxic to human bodies and 
therefore are non-environment friendly materials. One more important point is that the dielectric constant $(\varepsilon)$ for most of these ferroelectric ceramics has strong temperature dependence and shows rapid change near ferroelectric transition temperature which makes them inefficient to act as a good ceramic capacitor material. In the first of this millennium Subramanian et al. [15] have discovered a new pseudo-perovskite $\mathrm{CaCu}_{3} \mathrm{Ti}_{4} \mathrm{O}_{12}$ (CC TO) possessing a very high value of dielectric constant ( $\sim 10,000$ for bulk and $\sim 10^{5}$ for single crystals) and moderately lower dielectric loss $(\sim 0.1)$. The dielectric constant remains reasonably invariant over a wide temperature range from 100 to $400 \mathrm{~K}$ below $1 \mathrm{MHz}$. Hence the material is capable of potential industrial application for miniaturizing electronic devices. To a surprise the material is non-ferroic and shows a centrosymmetric structure down to $35 \mathrm{~K}$ [16]. Both intrinsic and extrinsic mechanisms were employed to explain this unusual phenomenon. Most of the studies have taken the help of Internal Barrier Layer Capacitor (IBLC) model consisting of semiconducting grains and insulating grain boundaries to explain this enormously large dielectric const [17-18]. whereas Lunkenheimer et al. believe it is arising from the electrode polarization effects [19]. Although there are few reports on the synthesis of nano-particles of this giant dielectric material [20], fabrication of its nanostructures is rare in literature. In this article we are reporting the synthesis of gaint dielectric CCTO nanotubes of about $200 \mathrm{~nm}$ diameter by means of a sol-gel method utilizing nano-porous anodic aluminum oxide (AAO) templates, structural characterization of the nano-tubes and high resolution electron microscopic (HRTEM) studies on the nano-tube walls to reveal the crystalline structures.

\section{EXPERIMENTAL DETAILS}

$\mathrm{CaCu}_{3} \mathrm{Ti}_{4} \mathrm{O}_{12}$ precursor-solution was prepared through conventional sol-gel route whreby metallic-acetates namely copper-acetate monohydrate $\left(\mathrm{Cu}\left(\mathrm{CH}_{3} \mathrm{COO}\right)_{2} \cdot \mathrm{H}_{2} \mathrm{O}\right.$ $>99 \%$, Aldrich) and calcium acetate monohydrate $\left(\mathrm{Ca}\left(\mathrm{CH}_{3} \mathrm{COO}\right)_{2} . \mathrm{H}_{2} \mathrm{O}>99 \%\right.$, Aldrich) were used as metal-ion precursors. Appropriate amount of these metallic-acetates, corresponding to $1: 3$ ratios of $\mathrm{Ca}: \mathrm{Cu}$ metal ions were dissolved in glacial acetic acid which was used as solvent. Titanium isopropoxide (TIP) (Ti- $\{\mathrm{OCH}-$ $\left.\left(\mathrm{CH}_{3}\right)_{2}\right\}_{4}>99.9 \%$, Aldrich) was used as Ti-source and was dropwise added in appropriate amount (i.e., amount corresponding 1:3:4 stoichiometric ratios of $\mathrm{Ca}: \mathrm{Cu}: \mathrm{Ti}$ metal ions) to glacial acetic acid with a gas-tight syringe while stirring the solution continuously with the help of a magnetic stirrer. It is a well known phenomenon that transition metallic alkoxides are unstable due to the presence of extremely reactive alkoxide groups and are prone to hydrolysis. Hence acetylacetone $\left(\mathrm{CH}_{2}\left(\mathrm{CH}_{3} \mathrm{CO}\right)_{2}\right.$, Merck) was added to the solution for stabilization of TIP. Next these two solutions were mixed together to form the final precursor solution which was adjusted to a concentration $0.1 \mathrm{M}$ (in terms of CCTO) and was subsequently refluxed for one hour at $160^{\circ} \mathrm{C}$ for homogeneity. The final precursor solution was transparent, dark blue in color and was stable for long time without any precipitation.

Nanopourous Anodic Aluminum Oxide (AAO) membranes (pore diameter: $\sim 200 \mathrm{~nm}$, and $60 \mu \mathrm{m}$ thickness) were purchase from Whatman $\AA$, were used as the templates for synthesis of CCTO nanotubes. Before using, templates were cleaned for $20 \mathrm{~min}$ in an ultrasonic bath using solvents of different polarity such as double distilled water, acetone, hexane, toluene and were annealed at $200^{\circ} \mathrm{C}$ for $2 \mathrm{~h}$ in vacuum to make free of any existent impurities. Next the AAO templates were immersed in the precursor solution for $2 \mathrm{~h}$ and were dried at $150^{\circ} \mathrm{C}$ for $1 \mathrm{~h}$. These templates containing the precursor were heated in air at $750^{\circ} \mathrm{C}$ for $1 \mathrm{~h}$ with the help of a thermal annealing furnace for the desired phase to form. CCTO nanotubes were isolated on removal of the AAO templates by immersing them in $6 \mathrm{M} \mathrm{NaOH}$ solution at room temperature for $24 \mathrm{~h}$. To obtain higher amount of nanotubes, the base solution was diluted in several steps with distilled water and finally with ethanol. The isolated nanotubes were collected by centrifugation. The structural studies on the as prepared CCTO nanotubes were carried out using X-ray powder diffraction (XRD) using a Philips PW3710 diffractometer $(\mathrm{Cu}-\mathrm{K}$ radiation, $30 \mathrm{kV}$ and $20 \mathrm{~mA}, \lambda=1.5406 \AA$ ). The morphology of the CCTO nanotubes was studied by scanning electron microscope (SEM) (Quanta 200). To reduce the charging effects on insulating AAO templates and CCTO nanotubes during SEM studies, a very thin layer of gold was sputtered for conductivity enhancement. The X-ray structural data were corroborated by the transmission electron microscopic (TEM, Tecnai F30) studies, operating at an acceleration voltage of $200 \mathrm{kV}$.

\section{RESULTS AND DISCUSSIONS}

Since the size and uniformity of the product nanotubes solely depend on the used nanoporous AAO template, its features namely the uniformity of size of the pores in the template were studied by SEM. Figure 1(a) and Figure 1(b) show typical scanning electron micrographs of top-view and lateral-views of an empty AAO template respectively. It can be noticed that the pores size (diameter) in the AAO template were in the range of 190$220 \mathrm{~nm}$ and pores are well-uniformly distributed over 
the template matrix in an approximately hexagonalshaped "honey-comb" like structure. Figure 1(c) shows SEM image of CCTO nano-tubes after almost $90 \%$ removal of the AAO template with $\mathrm{NaOH}$ solution. Figure 1(d) features a side view of some of the prepared nanotubes. The micrographs clearly show that as synthesized CCTO nanotubes were hollow, roughly parallel to each other, and vertically oriented on the AAO template to form an array. The average diameter of the nano-tubes are in the range of $160-180 \mathrm{~nm}$, little smaller than the expected value of $200 \mathrm{~nm}$. This contraction of nanotubes from the original dimension of the pores is probably due to the shrinkage experienced by CCTO nanotubes on densification and dehydration associated with thermal treatment. The diameter is quite uniform for most of the nano-tubes indicating the superiority of this method for large scale homogeneous nanotube synthesis.

To examine crystallinity and phase-purity of as-prepared nano-tubes obtained after annealing at $700^{\circ} \mathrm{C}$, $\mathrm{X}$-ray diffraction studies were carried out. Figure 2 shows the X-ray diffraction pattern of template free CCTO nano-tubes, which were collected and gathered as centrifuge residue after removal of $\mathrm{NaOH}$ solution and subsequent washing with different solvent mediums for several times. The XRD pattern clearly indicates the crystallinity of the nano-tubes. All the peaks in the diffractogram can be well-indexed according to the pseudoperovskite phase of CCTO (JCPDS card No. 75-2188). Absence of any impurity peaks proves the phase-purity. The orientations and the intensity ratios of the CCTO nanotubes matched well with the cubic pseudo-perovs-

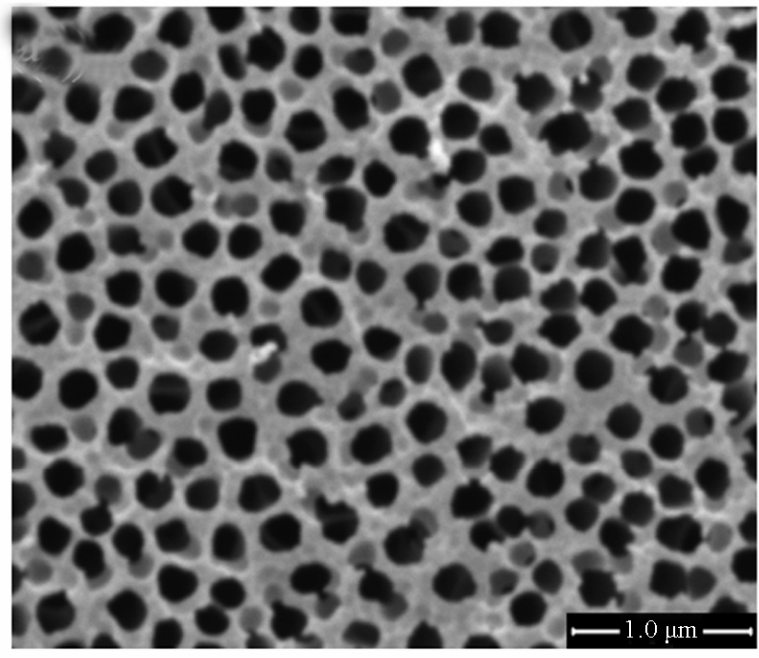

(a)

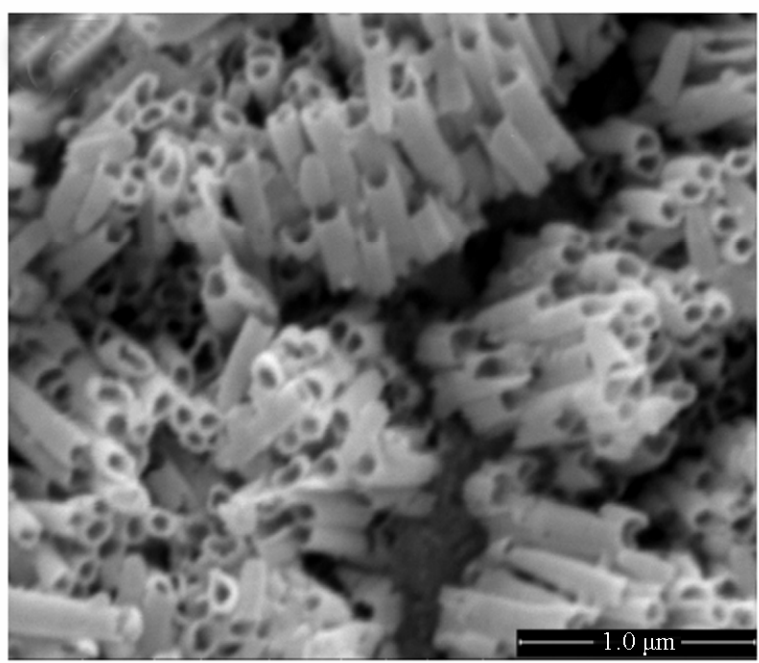

(c)

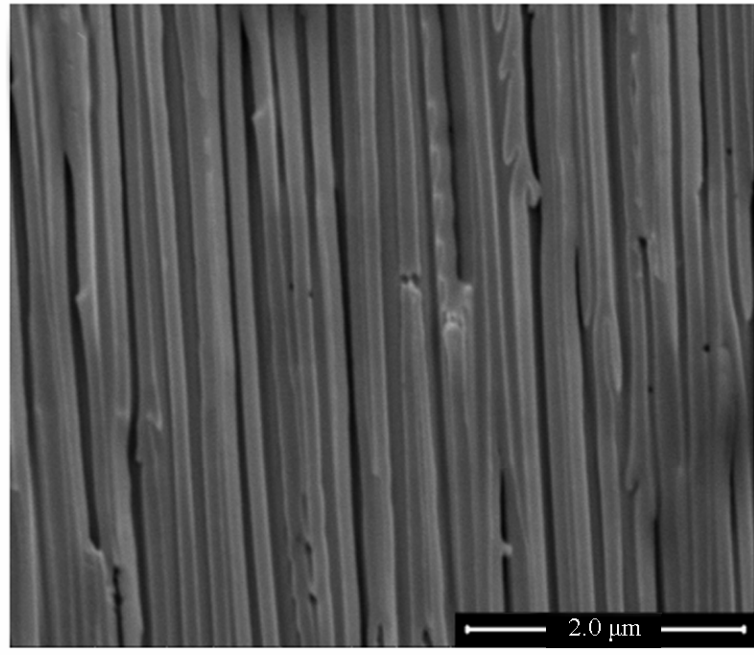

(b)

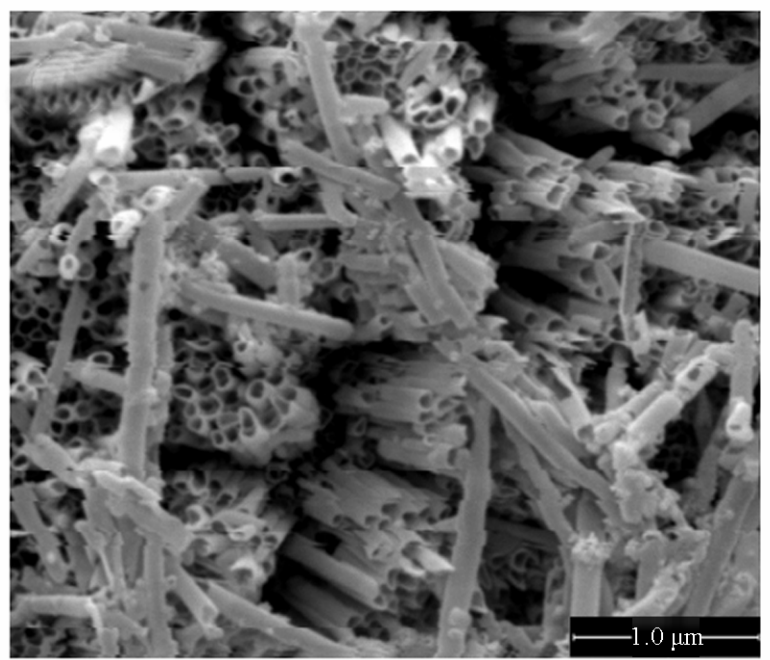

(d)

Figure 1. SEM images. (a) top-view of AAO template; (b) lateral-view of AAO template; (c) CCTO nanotubes after $>90 \%$ removal of AAO templates with the help of NAOH solution; (d) lateral view of some of the CCTO nano-tubes. 


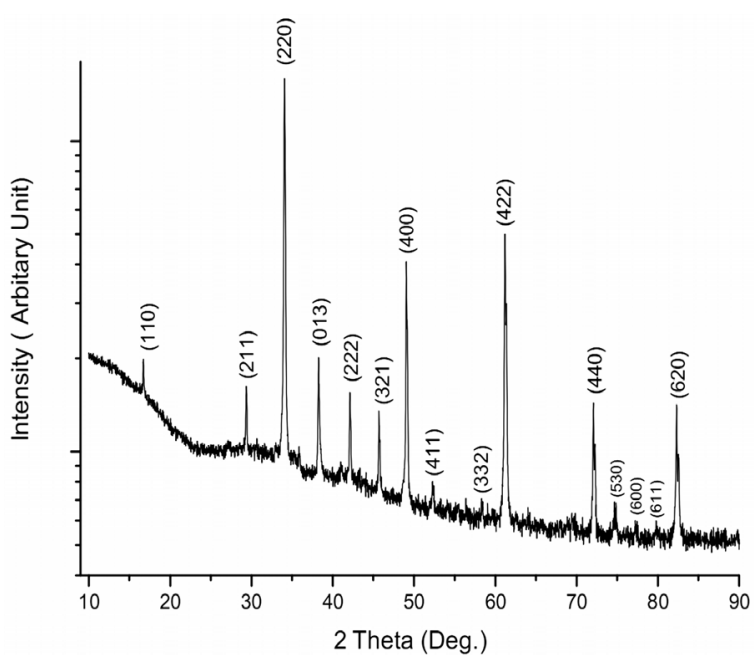

Figure 2. X-Ray Diffraction pattern of prepared CCTO nanotubes annealed at $700^{\circ} \mathrm{C}$ after removal of AAO templates.

kite phase of bulk CCTO.

Figure 3(a) shows TEM image of a CCTO nano-tube after complete removal of AAO template by dissolution in $6 \mathrm{M} \mathrm{NaOH}$ solution. The difference in contrast between the middle and the edges of the tube in Figure 3(a) clearly indicates its hollow structure. Enhanced scattering at edges of the tube makes it darker than the middle portion in the TEM picture. The diameter of the nano- tube is almost uniform throughout its length which is approximately equal (slightly smaller) to channel diameter of the applied template. The length of these nano- tubes was found to be shorter than the thickness $(60 \mu \mathrm{m})$ of the AAO templates. The possible reason may be the inefficient filling upto the pore-depth in the template and also breakage of some tubes to shorter ones during intense ultra-sonic dispersion treatment. Figure 3(b) re- presents corresponding SAED pattern which clearly proves the polycrystalline nature of the nano-tubes. The rings in the SEAD pattern corresponds to (422), (400), (013), (220) and (200) crystal planes of cubic pseudo- perovskite phase of CCTO. The d-spacings were calculated from the diameter of the bright circular rings observed in the electron diffraction pattern which were found to be in good agreement with that calculated from the XRD data (JCPDS card No. 75-2188) and the data available in the literature.

The micro-structure of the CCTO nano-tubes were further investigated with the help of High Resolution Transmission Electron Microscope (HRTEM) studies to get a clear insight of the features. Figure 3(c) shows a typical HRTEM image of the wall of a CCTO nano-tube prepared with the help of AAO templates. The HRTEM image clearly shows that the nano-tube is made up with a number of CCTO well-resolved nano-particles that are randomly oriented in the wall. The nano-particles comprising the walls of nano-tubes of other oxide materials have been previously observed and reported in the literature $[14,21]$. The size of the constituent particles is in the range of 5-10 nm which is in good agreement with the previously reported data of these functionally complex oxide nano-structures [14]. The observation of few unresolved spaces in the HRTEM image suggests the presence of few pores in the wall of CCTO nano-tubes. The lattice spacing values $(\mathrm{d} 220, \mathrm{~d} 400)$ as obtained from the HRTEM image closely resembles to that calculated on the basis of XRD and SEAD results.

Energy dispersive X-ray (EDX) spectrum of the CCTO nano-tubes after complete removal of AAO template is shown in the Figure 3(d). No emission peaks from $\mathrm{Al}$, and other elements from the etching $\mathrm{NaOH}$ solution, reagent, and template appeared in this spectrum. The absence of any impurity peaks in the EDS spectrum and the close resemblance of the atomic ratios of $\mathrm{Ca}, \mathrm{Cu}$ and $\mathrm{Ti}$ to the nominal composition in $\mathrm{CaCu}_{3} \mathrm{Ti}_{4} \mathrm{O}_{12}$ prove the elemental and phase purity of prepared CCTO nanotubes.

CCTO nanotubes were grown by sol-filling in the AAO template with the help of capillary force. The sol was drawn up into and filled the pores of the AAO template. Air in the pores and vapor from the sol were evaporated from the top surface of the AAO template. The possible formation mechanism of CCTO nanotubes inside the pores of AAO template is proposed here. Schematics of the possible formation mechanism of CCTO nanotube inside the pores of AAO template is shown in Figure 4 (growth process of CCTO nanotube). Initially, the template was put on the CCTO precursor solution as shown in Figure $\mathbf{4}$ because the solution is easier to fill into the pores of the template due to the capillary force. As the solvent evaporates from the surface and the concentration is enriched at the top of the pores, precipitation or gelation occurs first at the top of the pores on the AAO template surface exposed to the air and subsequently proceeds downward along the entire wall of the pores. The precursor solution consists of sol nanoparticles or nanoclusters homogeneously dispersed in the solvent. Such nanoclusters, which develop surface charges and form a double layer, can respond to an externally applied electric field or other nanoclusters [22]. When the surface of the AAO template is brought into contact with a sol, a surface charge and a double layer are formed on the surface of the AAO template. Therefore, when sols are drawn into the pore channels of the AAO template, if the surface charges of nanoclusters in a sol and the surface of the AAO template are opposite, there will be an electrostatic attraction force, and the 


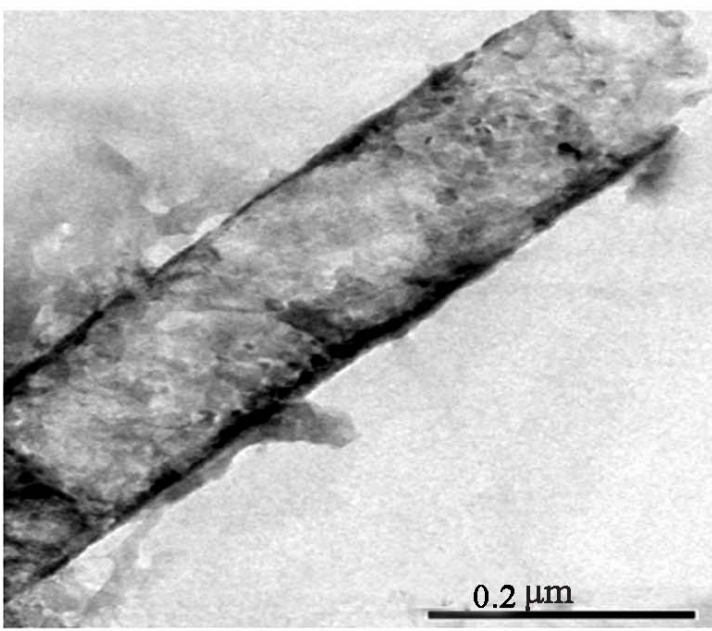

(a)

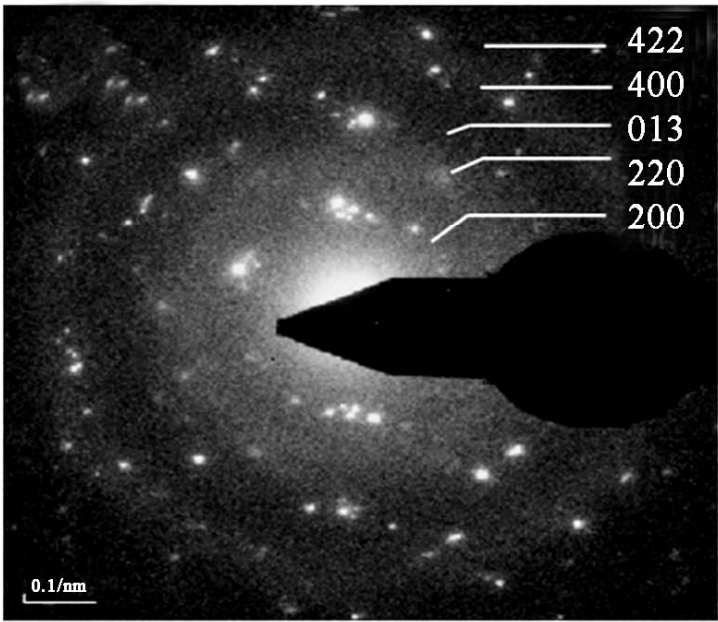

(c)

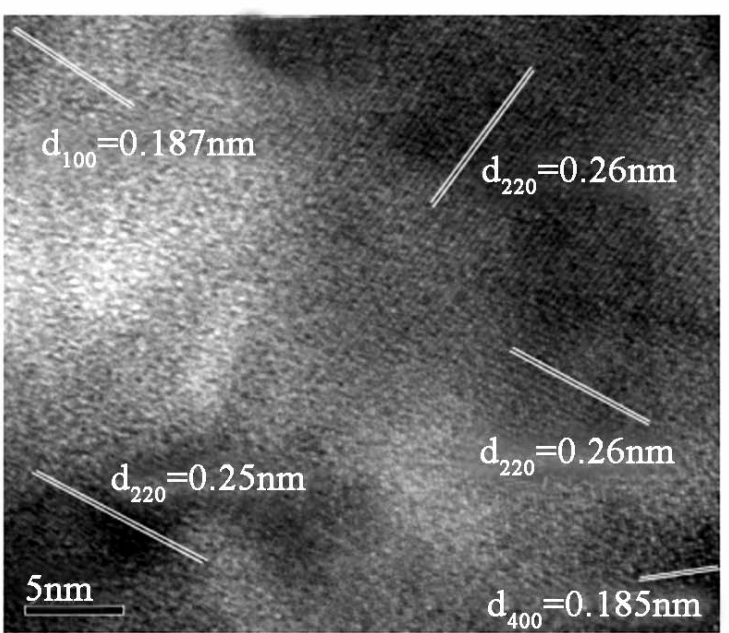

(b)

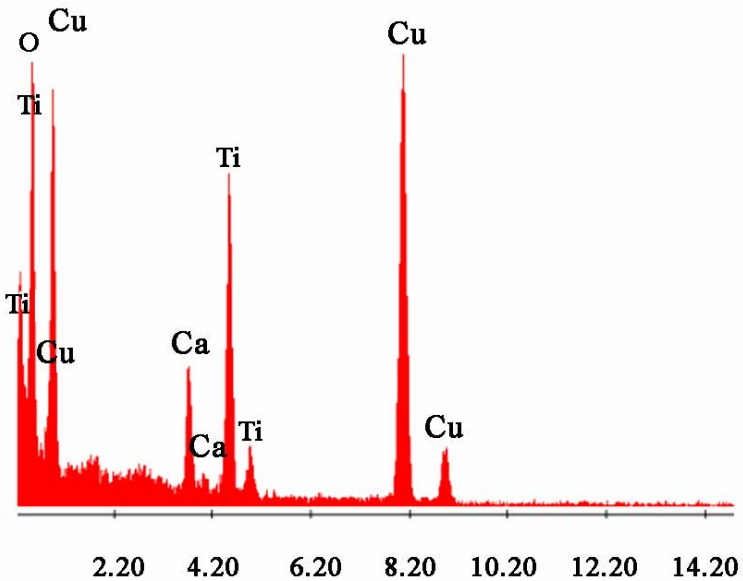

(d)

Figure 3. TEM image of (a) a CCTO nano-tube after complete removal of AAO template; (b) HRTEM image of a single nano-tube surface revealing that it is comprised of a number of randomly oriented particles; (c) corresponding SEAD pattern and (d) EDS spectrum of CCTO Nano-tubes.

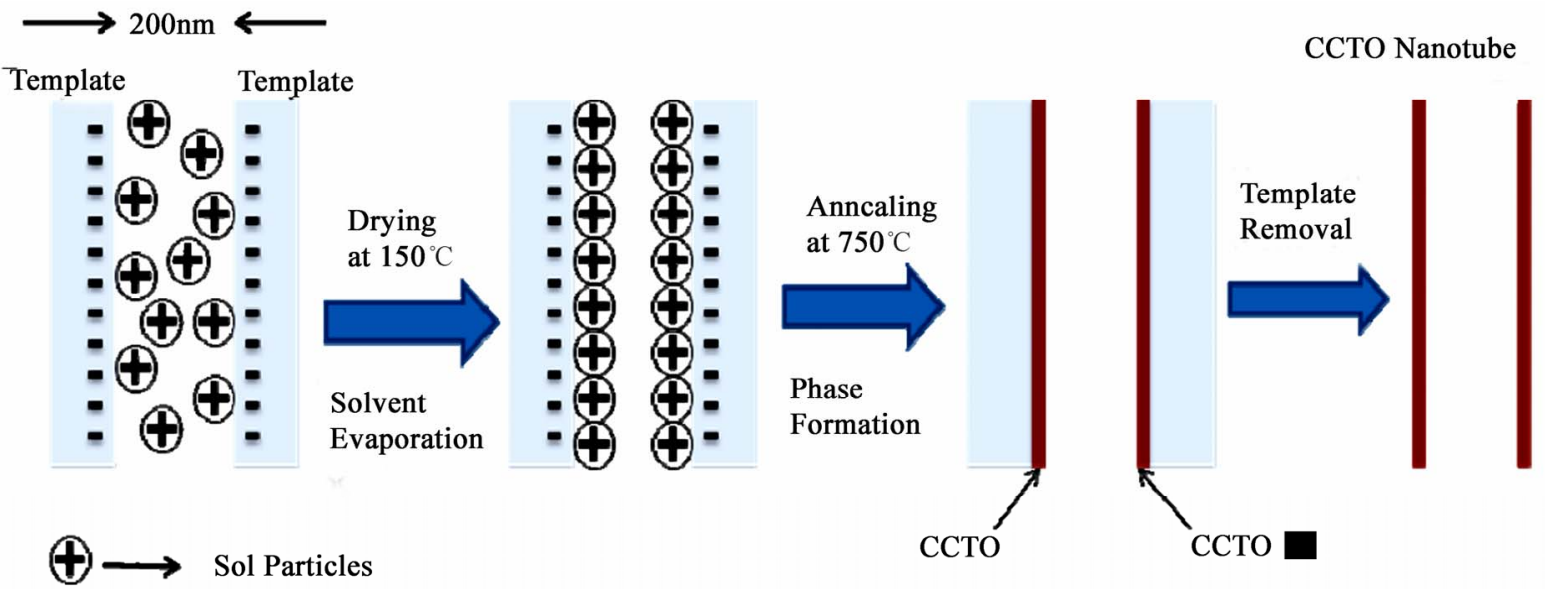

Figure 4. A schematic diagram of the possible growth mechanism of CCTO nanotubes in the AAO template. 
nanoclusters will preferentially deposit on the surface of the pore channel, resulting in the formation of nanotubes.

\section{CONCLUSIONS}

In summary, CCTO nanotubes have been prepared successfully by sol-gel method using the closely packed porous nanochannel alumina templates. The crystallinity and phase purity of the CCTO nanotubes were confirmed via XRD and SAED analysis. EDX analysis corroborated the stoichiometric $\mathrm{CaCu}_{3} \mathrm{Ti}_{4} \mathrm{O}_{12}$ formation. The walls of the nanotubes were found to be made up of nanoparticles as confirmed by the HRTEM studies. The nanoparticles embedded in the wall were found in the range of 5-10 $\mathrm{nm}$. This facile method of preparing the $\mathrm{CaCu}_{3} \mathrm{Ti}_{4} \mathrm{O}_{12}$ nanotubes at a large scale might be important for many applications in nanodevices.

\section{ACKNOWLEDGEMENTS}

N. B. thanks to Int. PH.D program, Indian Institute of Science for financial assistances and Institute Nano-Science Initiative for microscopic facilities.

\section{REFERENCES}

[1] Iijima, S. (1991) Helical microtubules of graphitic carbon. Nature, 354(6348), 56-58.

[2] Hu, J., Odom, T.W. and Lieber, C.M. (1999) Chemistry and physics in one dimension: Synthesis and properties of nanowires and nanotubes. Accounts of Chemical Research, 32(5), 435-445.

[3] Atzke, G.R.P., Rumeich, F.K. and Nesper, R. (2002) Based on oxide nanotubes and nanorods - anisotropic building blocks for future nanotechnology. Angewandte Chemie, 114(14), 2554-2571.

[4] Goldberger, J., He, R., Zhang, Y., Lee, S., Yan, H., Choi, H. and Yang, P. (2003) Single-crystal gallium nitride nanotubes, Nature, 422(6932), 599-602.

[5] Lee, S.B., Mitchell, D.T., Rofin, L.T., Ne vanen, T.K., Sçderlund, H. and Martin, C.R. (2002) Antibody-based bio-nanotube membranes for enantiomeric drug separations. Science, 296(5576), 2198-2200.

[6] Sha, J., Niu, J., Ma, X., Xu, J., Zhang, X., Yang, Q. and Yang, D. (2002) Silicon nanotubes. Advanced Materials, 14(17), 1219-1224.

[7] Junquera, J. and Ghosez, P. (2003) Critical thickness for ferroelectricity in perovskite ultrathin films. Nature, 422(6931), 534-539.

[8] Wang, Y. and Santiago-Aviles, J.J. (2004) Synthesis of lead zirconate titanate nanofibres and the Fourier-tran- sform infrared characterization of their metallo-organic decomposition process. Nanotechnology, 15, 32 .

[9] Luo, Y., Szafraniak, I., Zakharo, N.D., Nagarajan, V., Steinhart, M., Ehrspohn, R.B.W., Endorff, J.H.W., Ramesh, R. and Alexe, M. (2003) Nanoshell tubes of ferroelectric lead zirconate titanate and barium titanate. Applied Physics Letters, 83(5377), 440-442.

[10] Chu, M.W., Szafraniak, I., Scholz, R., Harnagea, C., Hesse, D., Alexe, M. and Gosele, U. (2004) Impact of misfit dislocations on the polarization instability of epitaxial nanostructured ferroelectric perovskites. $\mathrm{Na}$ ture Materials, 3(2), 87-90.

[11] Roelofs, A., Schneller, I., Szot, K. and Waser, R. (2002) Piezoresponse force microscopy of lead titanate nanograins possibly reaching the limit of ferroelectricity. Applied Physics Letters, 81(27), 5231-5233.

[12] Hernandez, B.A., Chang, K.S., Fisher, E.R. and Dorhout, P.K. (2002) Sol-Gel template synthesis and characterization of batio3 and pbtio3 nanotubes. Chemistry of Materials, 4, 480.

[13] Zhang, X.Y., Zhao, X., Lai, C.W., Wang, J., Tang, X.G. and Dai, J.Y. (2004) Synthesis and piezoresponse of highly ordered $\mathrm{Pb}\left(\mathrm{Zr}_{0.53} \mathrm{Ti}_{0.47}\right) \mathrm{O}_{3}$ nanowire arrays. Applied Physics Letters, 85(18), 4190-4192.

[14] Singh, S. and Krupanidhi, S.B. (2007) Synthesis and structural characterization of $\mathrm{Ba} 0.6 \mathrm{Sr} 0.4 \mathrm{TiO} 3$ nanotubes. Physics Letter A, 367(4-5), 356-359.

[15] Subramanian, M.A., Li, D., Duan, N., Reisner, B.A. and Sleight, A.W. (2000) High dielectric constant in $\mathrm{ACu}_{3-}$ $\mathrm{Ti}_{4} \mathrm{O}_{12}$ and $\mathrm{ACu}_{3} \mathrm{Ti}_{3} \mathrm{FeO}_{12}$ phase. Journal of Solid State Chemistry, 151(2), 323-325.

[16] Liu, J. J., Sui, Y.C., Duan, C.-G., Mei, W.-N., Smith, R.W., and Hardy, J.R. (2006) $\mathrm{CaCu}_{3} \mathrm{Ti}_{4} \mathrm{O}_{12}$ : Low-temperature synthesis by pyrolysis of an organic solution. Chemistry Materials, 18(16), 3878-3882.

[17] Sinclair, D.C., Adams, T.B., Morrison, F.D. and West, A.R. (2002) $\mathrm{CaCu}_{3} \mathrm{Ti}_{4} \mathrm{O}_{12}$ : One-step internal barrier layer capacitor. Applied Physics Letters, 80(12), 2153-2155.

[18] Liu, J., Duan, C., Yin, W., Mei, W.N., Smith, R.W. and Hardy, J.R. (2004) Large dielectric constant and maxwell-wagner relaxation in $\mathrm{Bi}_{23} \mathrm{Cu}_{3} \mathrm{Ti}_{4} \mathrm{O}_{12}$. Physics Review $B, 70,144106$.

[19] Lunkenheimer, P., Bobnar, V., Pronin, A.V., Ritus, A.I., Volkov, A.A. and Loidl, A. (2002) Origin of apparent colossal dielectric constants. Physics Review B, 66, 052105 .

[20] Thomas, P., Dwarakanath, K., Varma, K.B.R. and Kutty, T.R.N. (2008) Nanoparticles of the giant dielectric material, $\mathrm{CaCu}_{3} \mathrm{Ti}_{4} \mathrm{O}_{12}$ from a precursor route. Journal of Physics and Chemistry of Solids, 69(10), 2594-2604.

[21] Zhang, X.Y., Lai, C.W., Zhao, X., Wang, D.Y. and Dai, J.Y. (2005) Synthesis and ferroelectric properties of multiferroic $\mathrm{BiFeO}_{3}$ nanotube arrays. Applied Physics Letters, 87, 143102 .

[22] Wang, Y. and Cao, G. (2007) Synthesis and electrochemical properties of InVO4 nanotube arrays. Journal of Materials Chemistry, 17(2298), 894-899. 